\title{
Las transcripciones musicales del Baile Drama del Rabinal Achi
}

\author{
The musical transcriptions of Rabinal Achi Drama dance
}

Alfonso Arrivillaga-Cortés*, Matthias Stöckli

Area de Etnomusicología, Dirección General de Investigación, Universidad de San Carlos de Guatemala, Guatemala

*Autor a quien se dirige la correspondencia: laruduna@gmail.com

\section{Presentación}

Se ha seleccionado para la sección de documentos, este texto publicado originalmente en la Revista Tradiciones de Guatemala, No. 66 del Centro de Estudios Folclóricos, dedicada a la Etnomusicología en Guatemala (Stöckli, \& Arrivillaga, 2006).Se trata de un estudio que muestra el recuento de varias transcripciones musicales del Baile del Rabinal Achi, por cierto como podrá juzgar el lector, una de las primeras declaraciones impulsadas por Unesco como patrimonio de la humanidad. Siendo el presente número dedicado a los patrimonios intangibles que mejor que recoger una de las manifestaciones que caracterizan esta dimensión de los hechos culturales, y que, además como reflejan el estudio mismo, se expresan dinámicas. Dada la naturaleza de esta sección, se trascribe este documento en forma fiel al publicado originalmente, salvo el formato de la presente revista.

\section{Referencia}

Stöckli,M. \& Arrivillaga, A. (2006). Las transcripciones musicales del Rabinal Achi. Tradiciones de Guatemala, 66, 149-167. "en el vigésimo octavo día del mes de octubre del año 1850 transcribí el original de este baile del Tun, propiedad de nuestra villa de San Pablo de Rabinal, para dejar mí recuerdo a mis hijos y para que permanezcan con ellos de aquí en adelante. Amén" Bartolo Ziz ${ }^{1}$

En 1850, Bartolo Sis puso por escrito los diálogos del Rabinal Achi (o Xahoh Tun o Baile del Tun). Cinco años después se los dictaba al abate Charles-Étienne Brasseur de Bourbourg. Sin embargo, no fue sino hasta un año después, el 25 de enero de 1856, día de la Conversión de San Pablo, y tras mucha insistencia del abate que el baile-drama se representó por primera vez después de 30 años. Buscando que el registro de todo lo referente al baile se realizara, Brasseur de Bourbourg hizo que el maestro de capilla Nicolás "Colash" López y Vicente Tecú, ambos sirvientes suyos, anota-

1 Schwartz,1960. 
ran la música. ${ }^{2}$ Así se realizó la primera transcripción reconocida de música indígena a la que muchas otras iban a seguir, efectuadas en un inicio por viajeros y luego por estudiosos extranjeros y nacionales.

La partitura de López y Tecú, agregada como anexo a la traducción al francés del texto del Rabinal Achi por Brasseur de Bourbourg (1862), va a ser objeto de varias ediciones que la llevan a perpetuarse a lo largo del siglo XX dada su permanencia en publicaciones posteriores de este texto (por ejemplo: Cardoza y Aragón, 1929-30, 1953, 1972, 1975, 2001), en algunos compendios de partituras (Schwartz, 1960, 1981, 1987) y en artículos etnomusicológicos (Yurchenco 1978, 1985, 1990), convirtiendo esta en un fósil que no corresponde con la realidad dinámica de dicho evento. $^{3}$

No obstante, en septiembre de 1931, más de medio siglo después de dicha recopilación, Jesús Castillo presenció el baile en el Hipódromo del Norte de la ciudad de Guatemala (Castillo 1941, 1944) y lo transcribió a su vez. En su libro La música maya quiché fragmentos de esta transcripción le sirvieron para ilustrar sus análisis musicales. ${ }^{4}$

Cuando Henrietta Yurchenco recopiló la música del baile 14 años después, probablemente tocó todavía uno de los músicos del conjunto presenciado por Castillo. ${ }^{5} \mathrm{Su}$ transcripción es el resultado de un registro sonoro, por lo que el método empleado para efectuarla, difiere de sus antecesores. Llama la atención que esta transcripción la dio a conocer hasta 1985, después de la edición discográfica de 1978 en cuya libreta apa-

2 Las notas de Raynaud (1920) que aparecen como prefacio de la traducción de Luís Cardoza y Aragón del Rabinal Achi (1975, p. 9), dicen lo siguiente respecto de esa representación de 1856. “...la orquesta del Rabinal Achí solo comprendía dos trompetas y el tun (tunkul en Yucatán, teponaztli en México) o gran tambor sagrado. También tenían otros instrumentos de madera o de barro, como flautas (xul), silbatos de diferentes sonidos, calabazas huecas o llenas de granos o piedrecillas, con un mango para agitarlas o sirviendo de cajas de resonancia a un rudimentario instrumento de cuerda montado sobre una especie de arco, etc." Esta observación no queda muy clara con relación al instrumental utilizado en el baile mismo, dejando abierta la posibilidad de que más bien se trate de una descripción del ambiente festivo-musical que dio marco a este baile.

3 Además es de señalar que esta versión editorial original sirvió con mínimas modificaciones como facsímile para muchas de estas publicaciones.

4 Schwartz (1960) señala que en 1955 el baile se presentó por primera vez fuera de Rabinal, es decir, en la Antigua Guatemala así como en la ciudad de Guatemala, lo que contradice lo expuesto.

5 Se trata de Patrocinio Socop cuyo nombre Yurchenco indica como "P. Socub" (1978, p. 6). rece solamente un fragmento de la transcripción de López y Tecú.

En 1986, Enrique Anleu Díaz (Sacor, 1990) efectuó una nueva transcripción la que realizó al igual que Yurchenco con la ayuda de un registro magnetofónico. Dentro de esta misma línea Mark Howell (2004), etnomusicólogo norteamericano, recopiló el evento en mención en el 2002, y desarrolló su propia transcripción. ${ }^{6}$

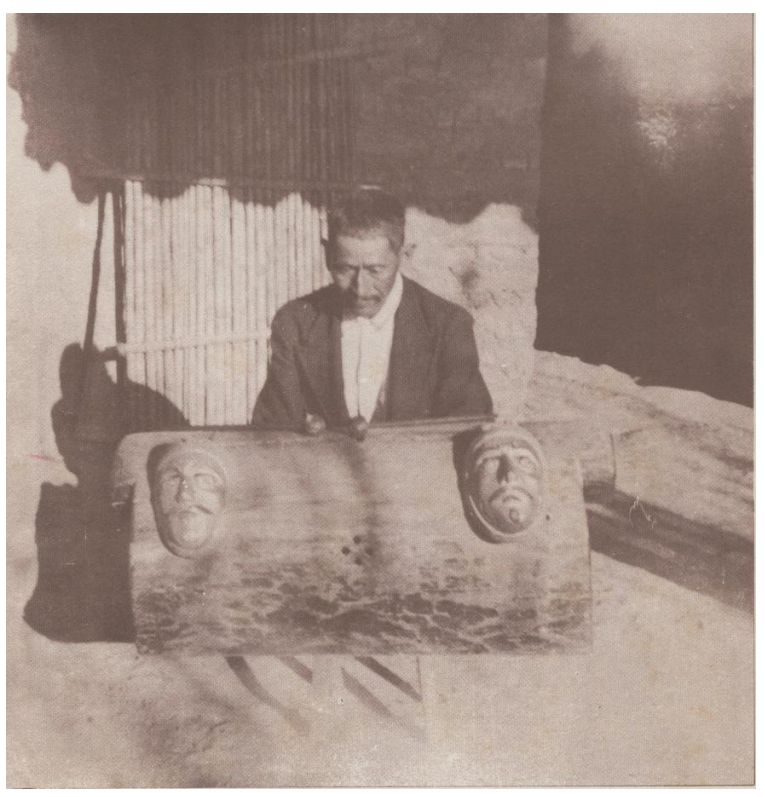

Figura 1. Esteban Xolop Sucup tocando el tun (Fotografía de Francisco Rodriguez Ruanet tomada del cuadernillo, Música de Guatemala No. 59, Schwartz, 1960).

\section{Una breve digresión}

El Baile del Rabinal Achi ocupa un lugar particular en la etnomusicología y etnocoreología de Guatemala. Es, entre otro, la tradición musical indígena con más fondo histórico, no solamente por sus supuestas raíces prehispánicas, sino también gracias a esta serie de transcripciones que acabamos de describir brevemente la cual permite seguir el desarrollo de la danza a través de cabalmente un siglo y medio. - ¿Lo permite? ¿No será que deberíamos dudar, junto a Henrietta Yurchenco (véase Homenaje), en la capacidad de las transcripciones de López y Tecú de representar

6 El archivo de fonogramas del área de etnomusicología del Centro de Estuidios Folkóricos de la Usac cuenta con copias y originales de las grabaciones de Yurchenco, Anleu Díaz y Fernando López. 
el modo como sonó la música en realidad en aquella famosa representación de la danza en la que el abate tanto insistió? ¿Y por lo tanto también en las de Casti1lo, efectuadas casi ochenta años más tarde, contrariamente a la afirmación de Yurchenco no idénticas con las de López y Tecú, pero compartiendo con ellas la representación supuestamente "occidentalizada" de una métrica estricta y convencional? No obstante, ¿a qué conclusiones sobre la práctica musical a mediados de los años cuarenta podemos llegar basándonos en la transcripción de Yurchenco misma, publicada en 1985 y representando obviamente un solo fragmento de toda la música del baile, verificable ahora sí, gracias a la grabación que se efectuó en el lugar, pero fragmentaria ésta a su vez y basándose en una sola toma? ¿Qué son las probabilidades que López y Tecú -familiares, como se debería suponer, tanto con las propiedades estructurales de la música indígena como con las de la música (eclesiástica) occidental- percibieron la música de la danza de una manera acertada, pero que no dispusieron ni de los conceptos ni de los medios gráficos precisos para representar su escucha de una forma adecuada? ¿O qué en realidad se tocaba en ese entonces la música del Rabinal Achi de un modo métrico mucho más estricto que noventa años después? ¿Qué sabemos sobre los modos de la transmisión de conocimientos y habilidades y sobre las probabilidades de cambio en una tradición musical que al parecer contaba con un ritmo de representaciones medido en décadas? - Son apenas algunas pocas preguntas acerca del estatus, la confiabilidad y la utilidad de transcripciones musicales como fuentes históricas ${ }^{7}$ cuya discusión más extensa, no obstante, tenemos que posponer hasta otra ocasión.

\section{Unas notas sobre el registro sonoro}

El registro sonoro por la vía de diversos soportes (inclusive la cámara de cine) merece el desarrollo de algunas líneas dado que se trata de una herramienta de documentación que evolucionó sensiblemente la etnomusicología y marcó sus derroteros a partir de los avances tecnológicos. La historia de la aplicación de estos aparatos en la recopilación de músicas de tradición oral es en América Latina tan larga como en

7 Se sobrentiende que semejantes preguntas también surgen al respecto de las demás transcripciones reproducidas en esta revista.
Europa. $^{8}$

Al parecer en Guatemala se inició esta historia con los registros en cilindros de cera que realizó Franz Termer durante su estancia en el país entre 1925 y 1929, “comisionada por la Sociedad de Geografía de Hamburgo" (Termer, 1957: xvi, véase también Heinitz, 1933). Poco tiempo después el registro en discos también se volvió una opción para esta actividad. Sin duda quienes trabajaron con este equipo realizaron una tarea formidable, como en el caso de Henrietta Yurchenco.

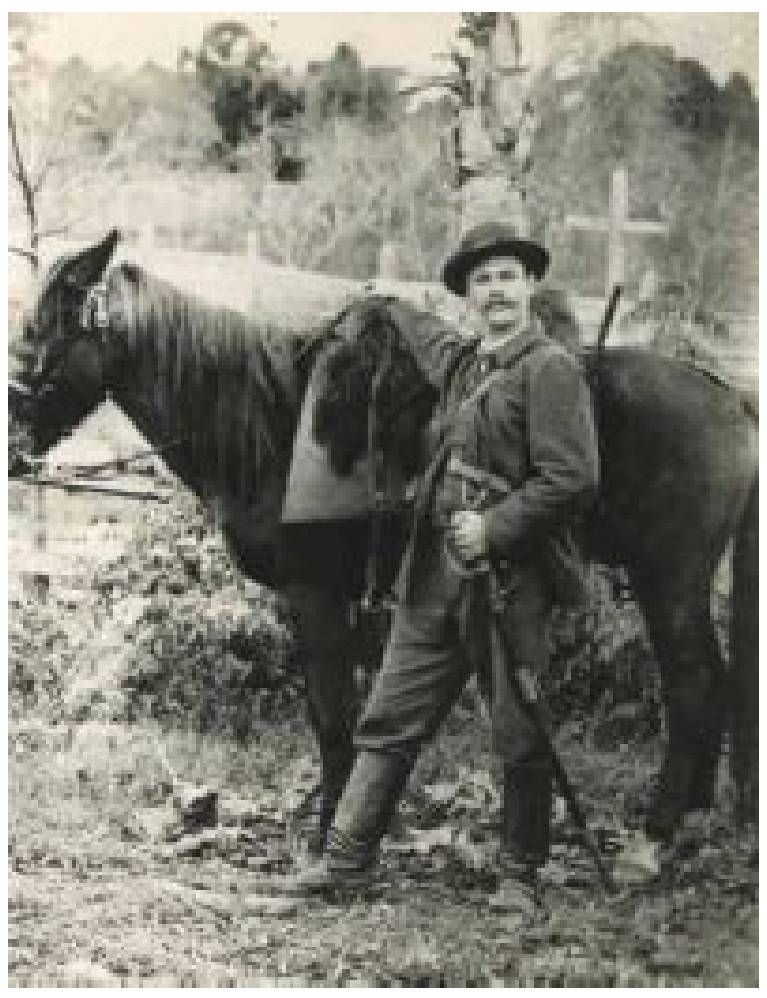

Figura 2. Franz Termer durante el trabajo de campo

El uso del grabador eléctrico de disco requería de mucha atención y habilidad. El grabador debía ir hilando la hebra del material del disco que se desprendía de su superficie al ser cincelado por la aguja que dejaba un surco con el registro a la medida que avanzaba. Como esta hebra se encontrará con la aguja se estro-

8 Véase Aretz $(1972,1991)$. Un detalle de la cantidad de registros existentes en cilindros de papel de estaño y de cera, así como de discos de vinilo sobre grupos indígenas del continente son referidos por Espinosa (2004, p. 11) con relación al proyecto inicial del Instituto Indigenista Interamericano (I.I.I.) para promover la música indígena. Un proyecto en el que se involucró Yurchenco (véase también Homenaje). 
peaba el registro que además era limitado en tiempo. La escasa infraestructura de estos países obligaba a viajar con un generador eléctrico complicando más el ejercicio del registro, $y$ haciendo mas pesado el equipo en su totalidad.

Cuando Lise Paret-Limardo de Vela realizó a principios de los años sesentas su labor, las grabadoras se habían desarrollado sensiblemente. Para entonces la cinta de carrete abierto (con posibilidad de varias velocidades) era la forma más común de registro, contando además con una buena calidad. Este tipo de grabadores también lograron reducir sus tamaños y se desarrollaron cintas metálicas (cromo, ferro-cromo, etc.) de alta calidad siendo la forma de registro más importante durante casi toda la segunda mitad del siglo XX.

Solo hasta finales del siglo XX, y más concretamente durante este siglo XXI, los equipos se redujeron sensiblemente en tamaño y peso, logrando ser más versátiles para el trabajo de campo. El registro a su vez pasó de análogo a digital modificando sensiblemente las formas de procesar el material.

\section{Bibliografía}

Aretz, Isabel. (1972). Colecciones de cilindros y trabajos de musicología comparada, realizados en Latinoamérica durante los primeros treinta años del siglo XX. En Revista Venezolana de Folklore, 2da. época No. 4, Instituto Nacional de Folklore, Caracas, pp. 49-65.

Aretz, Isabel. (1991). Historia de la etnomusicología en América latina: desde la época precolombina hasta nuestros días. Ediciones FUNDEF-CONAC-OEA, Caracas.

Brasseur de Bourbourg, Charles-Étienne. (1862). Rabinal-Achí ou le drame-ballet du Tun. En Collection de documents dans les langues indigènes, pour servir à l'étude de l'histoire et la philologie de l'Amérique ancienne..., vol. 2/ 2a parte. Arthus Bertrand \& Auguste Durand, Paris.

Cardoza y Aragón, Luis. (1929/30). Rabinal-Achí. El Varón de Rabinal. Ballet-Drama de los Indios Quichés de Guatemala. En Anales de la Sociedad de Geografia e Historia 6/ 1, pp. 45-51; 6/ 2, pp. 197-201; 6/ 3, pp. 347-370; 6/ 4, pp. 381-391.

Cardoza y Aragón, Luis. (1953). Rabinal-Achí. El Varón de Rabinal. Ballet-Drama de los Indios Quichés de Guatemala. Biblioteca de Cultura Popular
"20 de octubre" vol. 43. Ministerio de Educación Pública, Guatemala.

Cardoza y Aragón, Luis. (1972). Rabinal-Achí. El Varón de Rabinal. Ballet-Drama de los Indios Quichés de Guatemala. Editorial Porrúa, México. (Reedición: 1975).

Cardoza y Aragón, Luis. (2001). Rabinal-Achí. El Varón de Rabinal. Ballet-Drama de los Indios Quichés de Guatemala. Editorial Cultura. Ministerio de Cultura y Deportes, Guatemala.

Castillo, Jesús. (1941). La Música Maya-Quiche. Editorial Cifuentes, Quetzaltenango. Guatemala.

Castillo, Jesús. (1944). Legado folklórico a la juventud musical guatemalteca. Tipografía E. Cifuentes, Quetzaltenango.

Espinosa Velasco, Guillermo. (2004). Henrietta Yurchenco y el proyecto del I.I.I. sobre composiciones musicales contemporáneas con temas indígenas en los años cuarenta. En América Indígena 60/ 1, pp. 6-22.

Espinosa Velasco, Guillermo. (2004). Anexo de Henrietta Yurchenco y el proyecto del I.I.I. En América Indigena 60/ 1, pp.76-83.

Heinitz, Wilhelm. (1933). Chirimía- und Tambor-Phonogramme aus Nordwest-Guatemala. En Vox: Mitteilungen aus dem Phonetischen Laboratorium der Universität Hamburg 19/ 1-2, 1933, pp. 4-12.

Howell, Mark H. (2004). An Ethnoarchaeomusicological Investigation of Highland Guatemalan Dance-Plays. Tesis doctoral. City University of New York, New York.

Sacor Q., Hugo Fidel, et al. (1990). Rabinal Achi o Danza del Tun. Cuadernos de Investigación 1-90, Dirección General de Investigación, Universidad de San Carlos, Guatemala. (reeditado por: Colección Tierra Adentro 20, Subcentro Regional de Artesanías y Artes Populares, 1996).

Schwartz, Olga Vilma. (1960). Rabinal Achi. Música de Guatemala 59. Departamento de Recolección del Folklore, Dirección General de Bellas Artes, Guatemala.

Schwartz, Olga Vilma. (1981). Música de Guatemala. Universidad de San Carlos de Guatemala, Guatemala.

Schwartz, Olga Vilma. (1987). Música de Guatemala: Cuadernos de Folklore. Folklore, Dirección 
General de Bellas Artes. Folklore, Ministerio de Cultura, Guatemala.

Termer, Franz. (1957). Etnología y Etnografía de Guatemala. Seminario de Integración Social Guatemalteca 5. Editorial del Ministerio de Educación Pública, Guatemala.

Yurchenco, Henrietta. (1978). Music of the Maya Quiche of Guatemala. The Rabinal Achi and the Baile de las Canastas. Ethnic Folkways Records FE 4226, New York.

Yurchenco, Henrietta. (1985). The Rabinal Achí: A Twelfth Century Drama of the Maya-Quichés of Guatemala. En Acta Musicologica 57, pp. 37-50.

Yurchenco, Henrietta. (1990). El Rabinal Achí, un drama del siglo XII de los mayas-quichés de Guatemala. En Anales de la Academia de Geografia e Historia de Guatemala 64, 169-182.

\section{Las transcripciones}

1. Transcripción de Nicolás López y Vicente Tecú. Tomada de Brasseur de Bourbourg 1862.

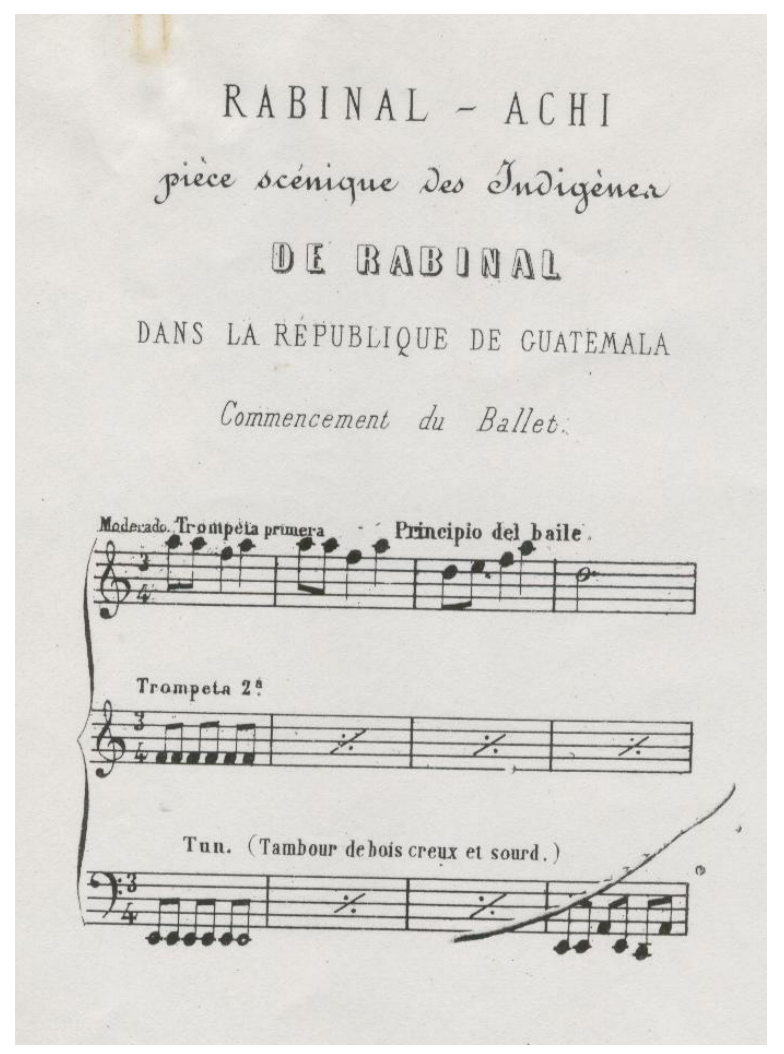

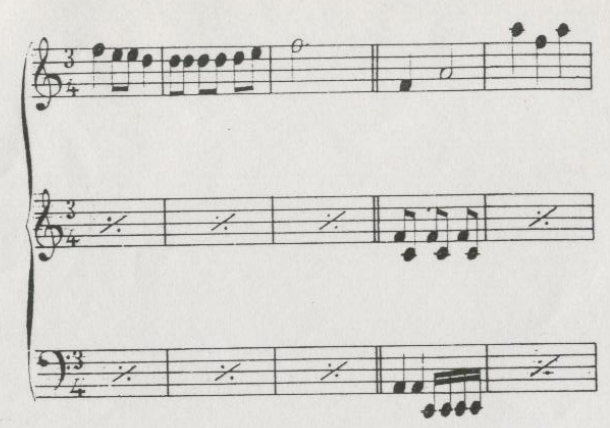
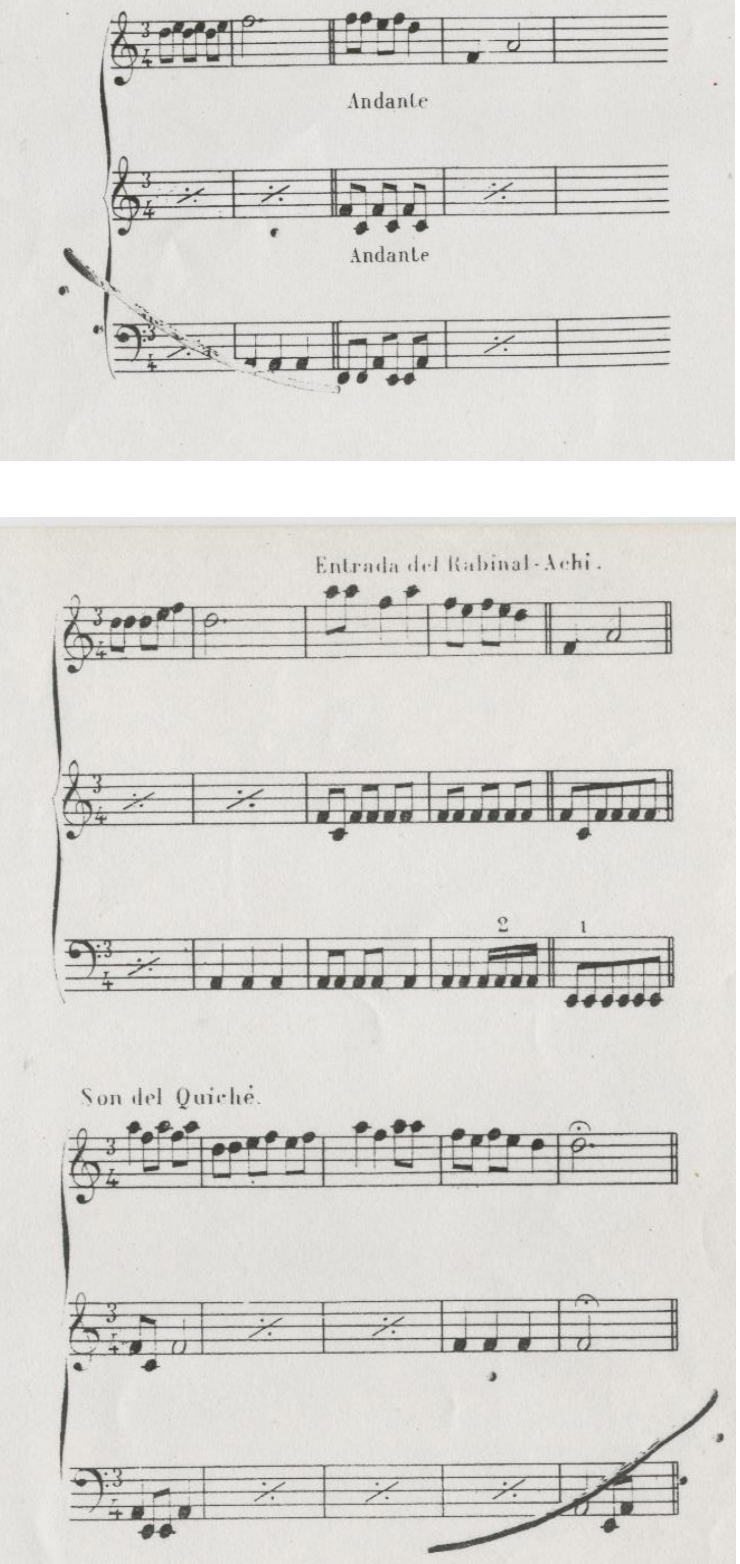

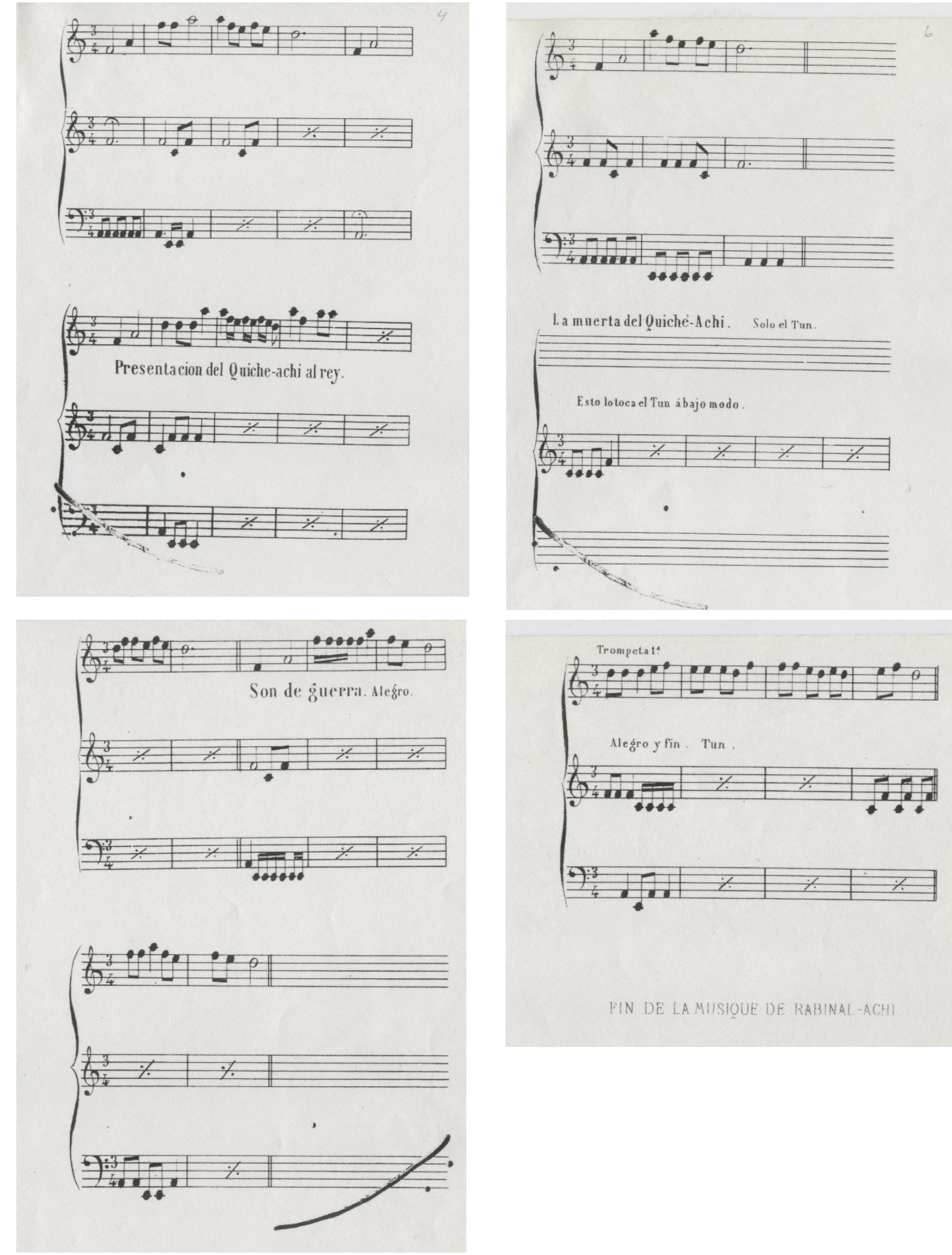

FIN DE LA MUISIOUE DE RABINAL-ACHI 
2. Transcripción de Jesús Castillo. Tomada de Castillo 1981.
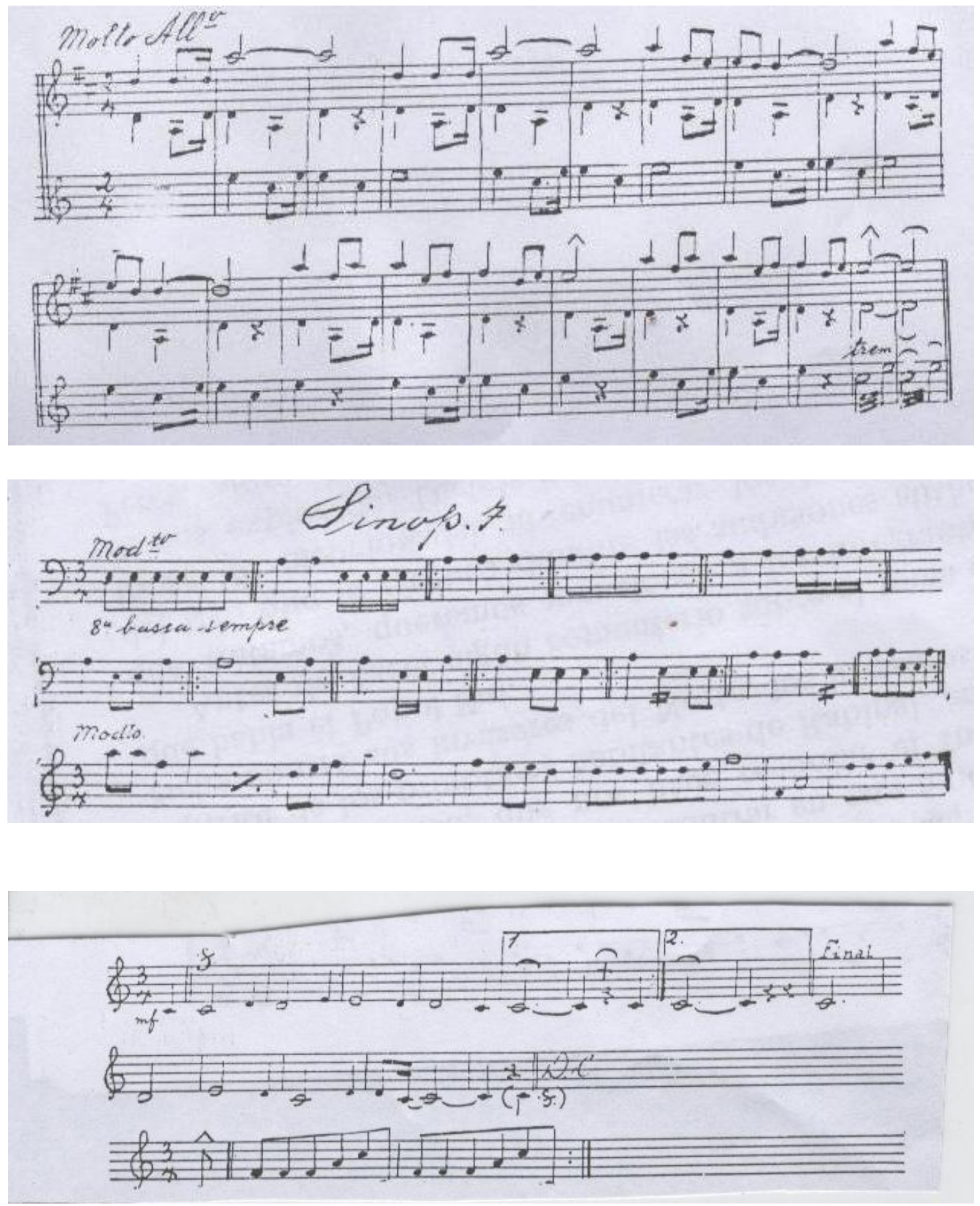
3. Transcripción de Henrietta Yurchenco. Tomada de Yurchenco 1985.

4. Transcripción de Enrique Anleu Díaz. Tomada de Sacor et al. 1990.

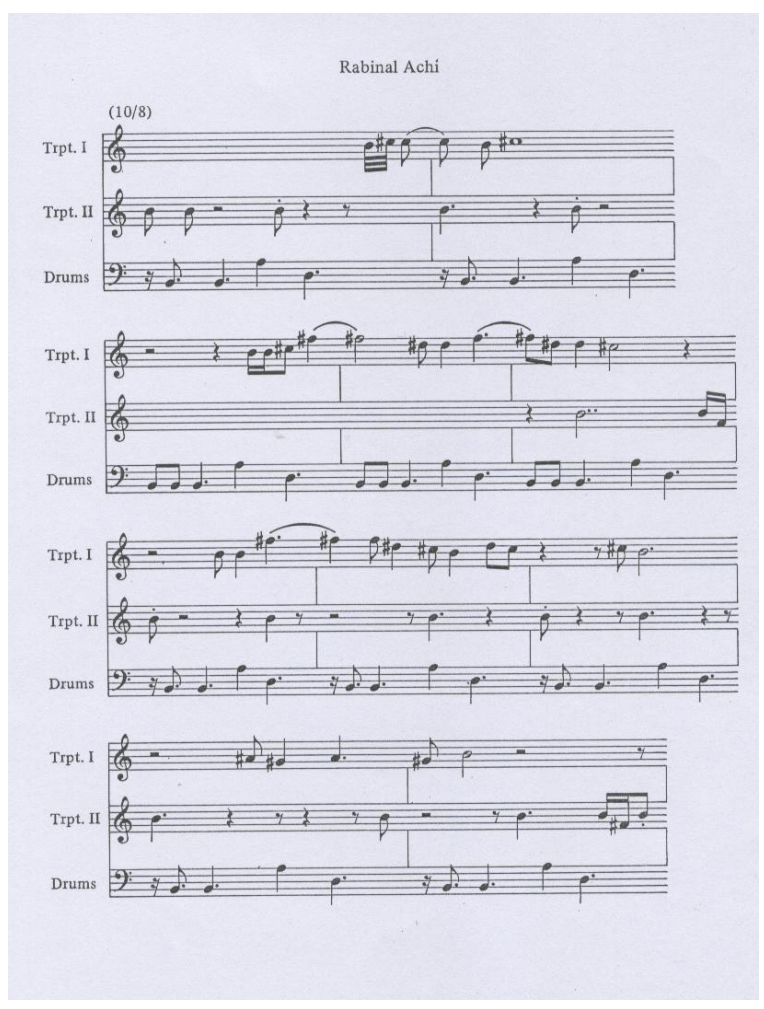

5. Transcripción de Mark Howell. Tomada de Howell 2004.
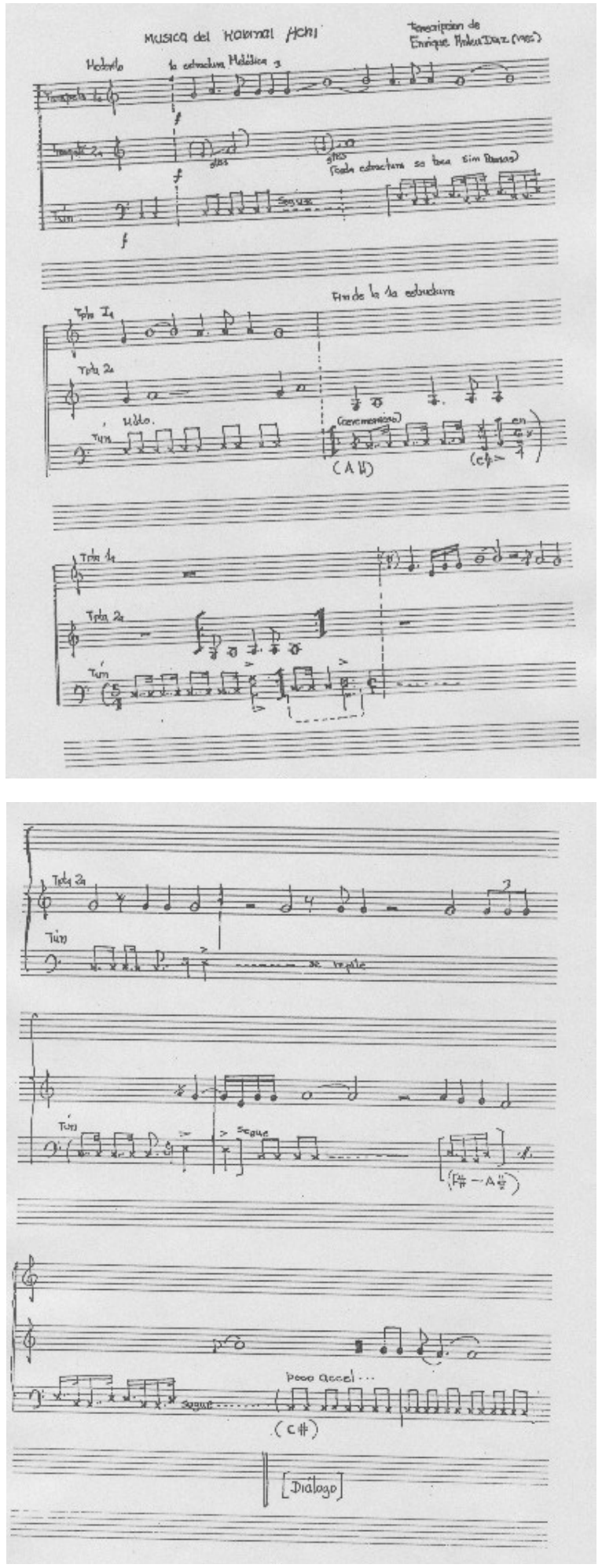


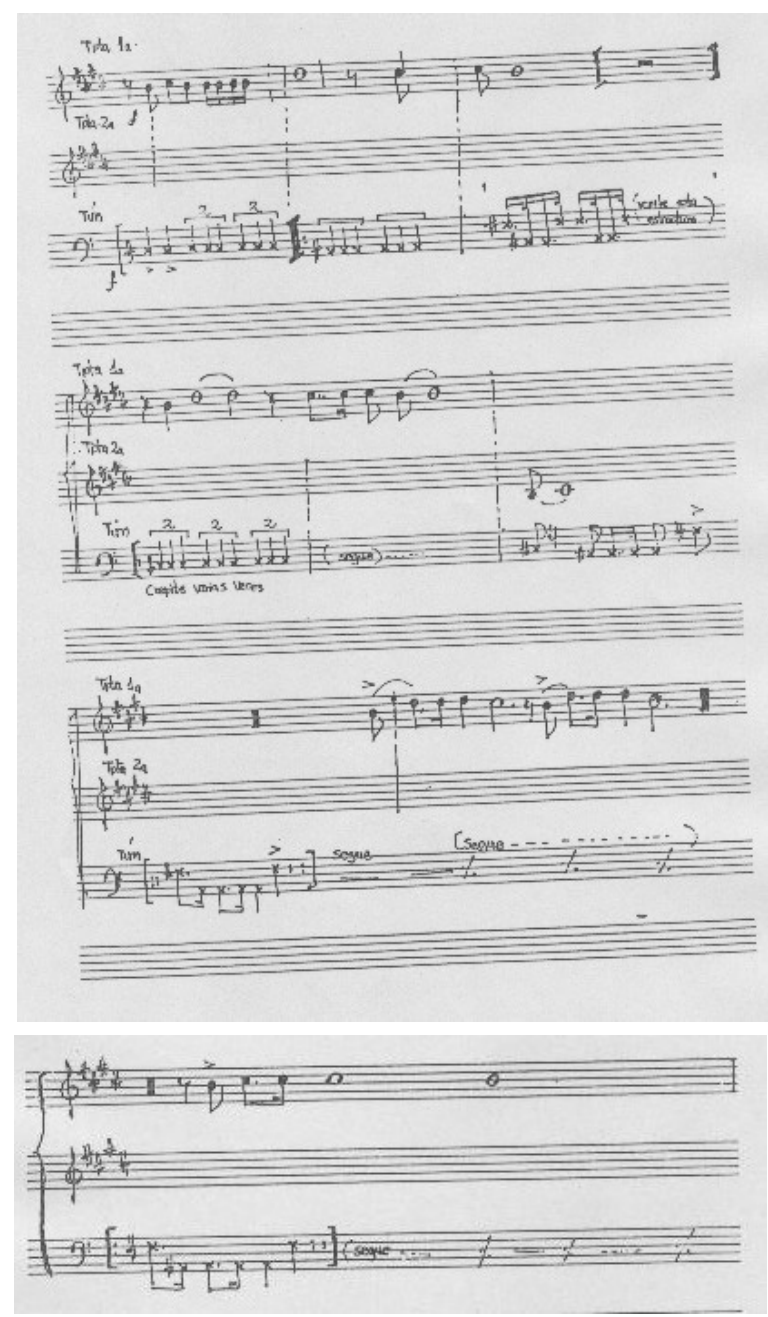

\section{Music Example 3}

The Template of the Rab'inal Achi Alto Performed on the Slit-Drum and Showing First and Second Trumpet Entrances, Ninth Piece, Calvaria Enactment in Rabinal,

$$
\text { January 23, } 2002
$$

Atto, 13 seconds (.13) in length

entrance of select parts indicated by timeline, relative durations of trumpet pitches indicated by use of

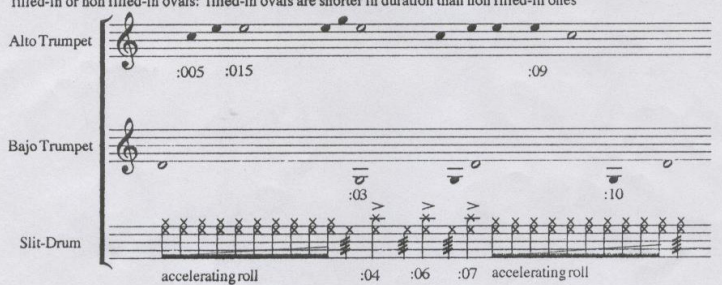

From Howell, 2002-Minidise Digital Recording, author's transcription
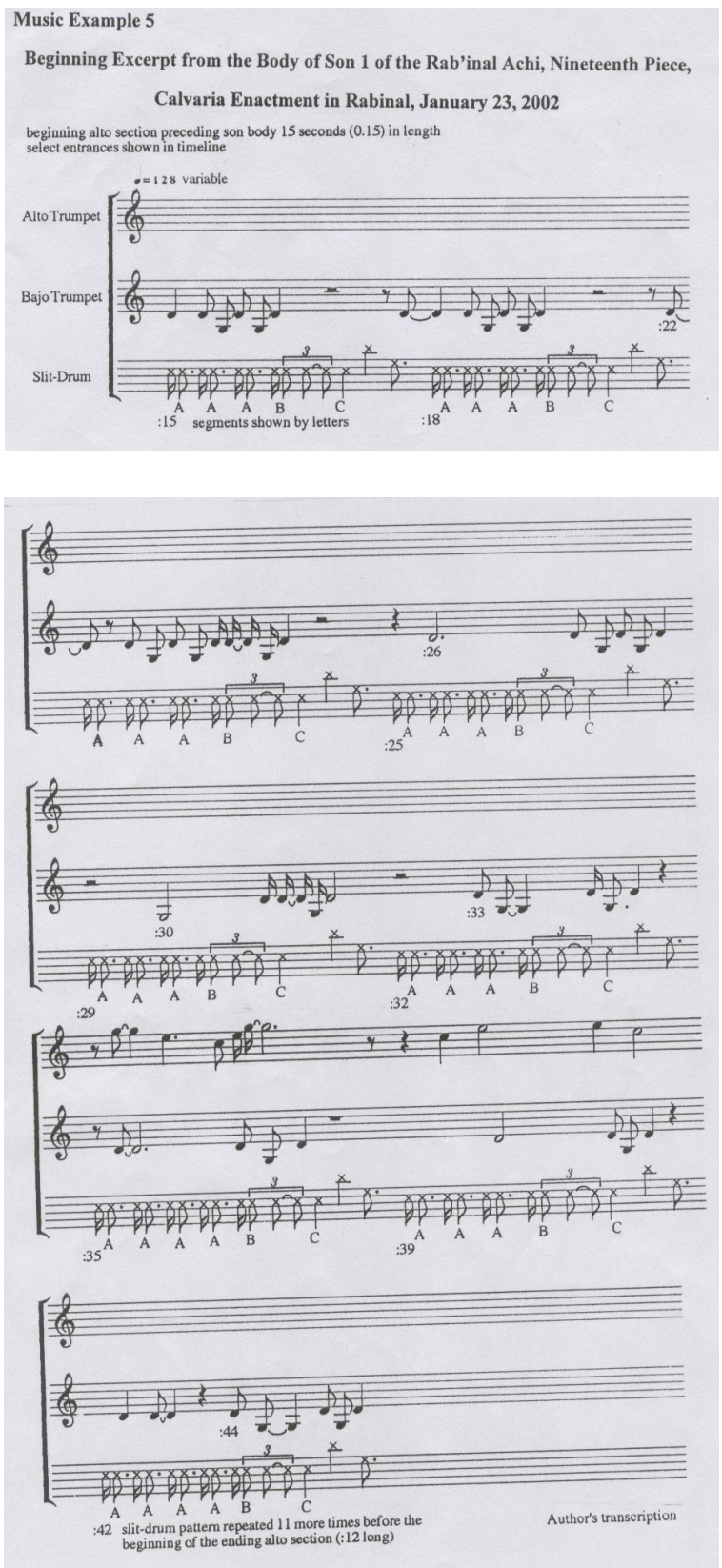